\title{
Summary of papers presented at the meeting of the Society for Social Medicine in Cambridge, September 1980
}

\begin{abstract}
Myocardial magnesium levels and IHD mortality
P. C. ELWOOD, P. M. SWEETNAM, W. H. B E A S LE Y, MRC Epidemiology Unit, Cardiff

The negative association between IHD mortality and water hardness is well known. Other studies have suggested that myocardial magnesium is lower in IHD than in non-IHD deaths and that it may be related to water hardness. Pathologists in 30 county boroughs in England and Wales were asked to take a one-centimetre cube of myocardium from 60 consecutive male necropsies.

The mean myocardial magnesium level was $167 \mu \mathrm{g} / \mathrm{gm}$. Magnesium levels were higher in sudden as opposed to lingering deaths, were lower in subjects with a previous illness, and decreased as time between death and necropsy increased. After allowing for all these factors, magnesium was $23 \mu \mathrm{g} / \mathrm{gm}$ lower in IHD deaths than in non-IHD deaths. It seems unlikely that myocardial magnesium levels are the explanation of the water story.
\end{abstract}

Myocardial infarction and oral contraceptives: has the risk changed?

M. THOROGOOD, S. A. ADAM, J. I. MANN, Department of Social and Community Medicine, University of Oxford

All deaths from myocardial infarction occurring during 1978 in women aged 15-44 in England and Wales were investigated. Using a case-control approach, oral contraceptives were found to be associated with an approximately twofold increase in risk of this condition (relative risk $2 \cdot 2: 1,95 \%$ confidence limits $1 \cdot 2-4 \cdot 2$ ) but the effect was apparent only when women with no known risk factors for ischaemic heart disease were considered separately. Preparations containing $30 \mu \mathrm{g}$ or less of oestrogen were associated with the same risk as those containing $50 \mu \mathrm{g}$, but this could be due to the fact that preparations containing less oestrogen tend to contain higher doses of progestagens.

Decision-making after myocardial infarction

T. W. DA VIES, A. SORENSEN, P. FARRELL, Department of Community Medicine, University of Cambridge

Admissions to hospital with myocardial infarction are constantly lower on Sundays than on Mondays. This could be caused either by a similar cycle of incidence, as the national figures for deaths suggest, or by patients delaying their calls for help, as most clinicians suggest. A study was carried out on all patients admitted to Addenbrooke's Hospital from February to June 1980 with acute myocardial infarction.

The number of admissions was lower on Sunday than on Monday. However, the onset of symptoms did not follow the same cycle, and there was a relationship between delay time (the time between onset and admission) and the times of onset and admission. It therefore seems that the cycle of admissions is the result of changes in patient behaviour. Men under 65 (or their wives) took longer to call the doctor, but this was not the cause of the longer delay at weekends.
The effects of health education on knowledge, attitudes, behaviour and risk factor levels in the United Kingdom Heart Disease Prevention Project

H. TUNSTALL PEDOE, R. HELLER, G. ROS E, Department of Epidemiology, St. Mary's Hospital, London

The effects of a health education programme on industrial workers were assessed by comparing risk factor levels in samples of men in intervention and control groups at two and four years, and after five to six years, when all remaining men in both groups were offered an examination accompanied by a questionnaire. Risk factor differences were greatest at four years, after an intensification of the campaign, than they were at two years or at the end, but the latter examination enabled a comparison to be made of health-related knowledge and attitudes on the two sides of the trial.

These seemed little affected. Slightly fewer men in the intervention group were worried about the risk of having a heart attack but the same proportion (75\%) as of controls thought that a man could reduce his chances of having one. The prominence given by the two groups to the many different alleged causes of heart attack was very similar, with a small percentage increase only on the intervention side in those blaming cigarettes, fat in the diet, and blood pressure, factors stressed in the campaign. However, major differences were apparent in self-reported behaviour in relation to diet, exercise, and smoking. The effect of the intervention seemed to be to modify behaviour rather than knowledge and attitudes.

\section{Community medicine in the Cambridge medical school}

R. M. ACHESON, Department of Community Medicine, University of Cambridge

The history of the Cambridge 'School of Physic' since the earliest days, and of the investment of John Addenbrooke's bequest (1719) in a hospital, were briefly reviewed. The new clinical medical school, and the Department of Community Medicine, both of which came into existence in 1976, were described.

Operational research in the health services

P. C. SMITH, Department of Community Medicine, University of Cambridge

There is considerable misapprehension about the nature and role of operational research. The philosophy of operational research, expressed by Ackoff, is 'designing a desirable future ... and inventing ways of bringing it about'. The planning process is illustrated by a strategic plan produced for the maternity services in Cambridge Health District, showing how the principles of participation, continuity and comprehensiveness can be applied in practice. The study method followed the familiar pattern of setting objectives and devising strategies to achieve those objectives. In contrast to most health planning, however, objectives were expressed in terms of the health of mothers and babies, and not provision of resources. The strategies were then examined in conjunction with 150 
constraints-population, policies and programmes, organisations' health methods, and resources - to produce a realistic long-term plan for the maternity services.

Computerised screening of radioisotope liver scans using statistical pattern-recognition techniques

R. H A N KA, L. P. HEN DERSON, Department of Community Medicine, University of Cambridge

Recent advances in technology have resulted in a dramatic fall in the cost of computing facilities, giving rise to many possible applications which were previously uneconomical and technically not feasible. One of the most interesting of these is the use of a computer as an aid to the physician in medical diagnosis of images.

A statistically based method for the classification of radioisotope liver scans was devised. Comparisons were made of the diagnostic accuracy of the computerised system and of both senior and junior medical staff, and the applications of computers for routine diagnostic tasks and preventive screening were considered.

Aspects of the epidemiology of colon cancer

D. R. R. WILLIAMS, Department of Community Medicine and Dunn Clinical Nutrition Centre, University of Cambridge

The role of dietary fibre in the aetiology of colon cancer has not been clearly established in epidemiological studies. There are difficulties in using case studies in testing the fibre hypothesis. The disease itself may influence present diet and the investigation of past diets by interview and questionnaire techniques has never been fully evaluated. In addition, nothing is known of the critical time at which dietary exposure is important for the subsequent development of colon cancer, of how to assess changes in diet in an individual's past life, and of the dietary fibre content of many foods.

Since the 'fibre hypothesis' suggests that faecal bulking is an important aspect of protection from colon cancer, the relationship of colon cancer mortality to pentose intakes (calculated from National Food Survey data) has been tested in standard regions of Great Britain. A negative correlation was demonstrated and no relationship was found with fat, protein, or total dietary fibre, though a relationship was demonstrated with pentose-containing vegetables.

Occupational and non-occupational factors in regional mortality

L. F. S A UGST A D, Institute of Occupational Health, Oslo

The municipalities in Finnmark county in northern Norway were grouped according to predominant occupation and mortality and cause of death in men aged over 20 in 1966-78. Age-adjusted rates were calculated using the 1970 census population.

The highest mortality was observed in the fishing industry in the towns and the rural areas, and the lowest where reindeer and service were the predominant occupations. Mortality from cancer (ICD 140-209) was 31\% above the mean in the towns, and respiratory cancer (ICD 160-4) mortality was 122 per 100000 , compared with 101 in the mining municipality, 41 in the service and reindeer area, and 78 in the county. There was no increased risk of lung cancer in the mining municipality. Among the towns, respiratory cancer mortality was 75 per 100000 in Hammerfest in Western Finnmark as against 145 in Vardø and Vadsø in Eastern Finnmärk.

It is suggested that the significant excess mortality from respiratory. cancer in both sexes in Vardø-Vads $\varnothing$ is the expression of the cumulative effect of an additional atmospheric pollution during the last $\mathbf{3 0}$ years. Excessive concentrations of trace elements, such as nickel, chromium, arsenic, copper and selen, in addition to excess sulphur and nitrogen, reaches these towns from industries in the USSR.

\section{Regional differences in survival from cancers}

A. J. SILMAN, S. J. W. EVANS, Department of Clinical Epidemiology, London Hospital Medical College Newly available data on cancer survival within regions in England and Wales were analysed to detect any differences in survival and to suggest possible explanations. There was a wide regional variation in five-year survival from most major cancers registered in 1971-3. Cumulative mortality curves showed that these differences were present one year after registration and persisted over the subsequent four years. The more lethal cancers had the largest relative differences in survival, with a fourfold difference between the best and worst regions. Conversely, the less lethal cancers had the largest absolute differences of up to $25 \%$. In general the metropolitan regions were better and regions in the north and north-west of England were worse than average. These regional differences are probably real rather than artefactual, the likely explanation being one or more of the following: late presentation of disease, level of general health affecting case fatality, and availability of medical intervention.

Regional variations in cardiovascular disease in Great Britain and the prevalence of risk factors

A. G. SHAPER, S. J. POCOCK, M. WALKER, N. M. COHEN, Department of Clinical Epidemiology and General Practice, Royal Free Hospital School of Medicine, London

The British Regional Heart Study aims to determine the factors responsible for the marked regional variations in cardiovascular disease. Twenty-five towns have been selected to represent the full range of cardiovascular mortality and water hardness and the major geographic regions. Some 8000 men aged 40-59 drawn from representative general practices in these towns are being studied to relate the prevalence of individual risk factors to cardiovascular mortality. A prospective study will relate incidence of cardiovascular events to individual environmental risk factors. Preliminary results from the first 16 towns are available.

On a town basis, mean levels for measured variables have been related to standardised mortality ratios for cardiovascular disease (1969-73). The data suggest that blood pressure, smoking, and alcohol intake are associated with inter-town differences and that blood cholesterol, HDL levels and body mass index are not. The interrelationships between these variables on an individual basis have been examined in 5000 men using multiple regression. Body mass index and alcohol intake are both strongly related to blood pressure. 
Incidence of diabetes in nine British towns

C. POWER, D. J. P. BARKER, M. J . GAR DNER, MRC Environmental Epidemiology Unit, University of Southampton

The incidence of diabetes was measured in nine towns in England and Wales. During 1977-8 the diabetic physicians in the towns maintained registers of all newly diagnosed diabetics aged 18-50. Registration criteria were either a random blood glucose value greater than $11.1 \mathrm{mmol} / \mathrm{l}$, or a fasting level greater than $6.7 \mathrm{mmol} / \mathrm{l}$, or a two-hour value greater than $10 \mathrm{mmol} / \mathrm{l}$ after $50 \mathrm{~g}$ of glucose; 254 cases were reported. The majority $(66 \%)$ were managed by diabetic drugs or diet alone; the remainder were treated by insulin therapy. Among the insulin-taking diabetics there was little association between the age-sex standardised incidence rates and either socioeconomic conditions or latitude. However, for the diabetics not taking insulin there was a markedly higher incidence in each of the three towns with the worst socioeconomic conditions. The average for the three towns in each socioeconomic group showed a progression from 10 per 100000 per year for the better towns to 13 for the medium and 23 per 100000 per year for the worst towns. Incidence of diabetes showed no latitudinal difference. The variation between the better and worse towns could not be explained by variations in physicians' prescribing habits, general practitioners' referral practices, or social class distribution.

Alcohol and mortality in British civil servants

M. G. MARMOT, G. ROSE, M. J. SHIPLEY, Department of Medical Statistics and Epidemiology, London School of Hygiene and Tropical Medicine

Several studies have shown that alcoholics have a higher mortality than the average. By contrast, the largest single component of mortality, cardiovascular disease, has been reported to be more frequent among non-drinkers than among drinkers. In 1926, Pearl reported that the curve relating alcohol consumption to mortality was U-shaped.

The relation between reported alcohol consumption and mortality was examined in the Whitehall study. One thousand four hundred and twenty-two male civil servants aged 40-69 completed a 24-hour dietary recall questionnaire subsequent to a screening examination. Mortality in the subsequent 10 years has been recorded. The mortality from all causes was over $30 \%$ higher in men reporting no alcohol consumption ('non-drinkers') than in men reporting 'moderate' alcohol consumption (1-34g alcohol/day). The mortality in 'heavy' drinkers, $(\geqslant 35 \mathrm{~g}$ alcohol/day) was the same as in 'non-drinkers', that is, the U-shaped curve of Pearl was observed. Cardiovascular mortality was higher in 'non-drinkers' than in 'moderate' or 'heavy' drinkers-with no clear difference between 'moderate' and 'heavy' drinkers. Non-cardiovascular mortality showed the U-shaped pattern. The highest mortality was in the 'heavy' drinkers, among whom the age-adjusted mortality was over 2.5 times that of the 'moderate' drinkers and over 1.5 times that of 'non-drinkers'. Controlling for smoking, blood pressure, plasma cholesterol, and grade of employment did not reduce the size of the association.
Prevalence of urinary tract infection symptoms in women M. WALKER, J. A. HEA DY, A. G. SHAPER, Department of Clinical Epidemiology and General Practice, Royal Free Hospital School of Medicine, London

General practitioners recognise urinary tract infection in women as a common and frequently recurring problem. It may lead in a minority to chronic kidney disease. A postal survey was carried out in four general practices in North London to measure the size of the problem and to relate prevalence to individual characteristics of the women before planning a detailed bacteriological study. Questionnaires were sent to 7700 women aged 20-54 and 4100 usable replies were received. Fifty-two per cent of the women recorded relevant symptoms at some time in their lives, $20 \%$ in the previous year, and $10 \%$ had had more than one episode in the last 12 months. Seventy-eight per cent of women récording symptoms had consulted their doctor at some time, but the vast majority of episodes were not reported. Contrary to expectation, prevalence varied only slightly with age, parity, marital status or social class. A relationship emerged between the early onset of the problem and frequent recurrent symptoms.

The wider role of an epidemiologist in disease prevention after investigation of a local outbreak of hepatitis B from tattooing

N. D. NOAH, Communicable Diseases Surveillance Centre, Colindale

After an outbreak of hepatitis B which was traced to a tattooist, the hygienic methods used by him were observed and detailed recommendations made for their improvement.

This led to an interest in the hygiene of tattooists, and, in conjunction with a tattooist organisation, a code of practice on hygienic tattooing was drawn up. This has been widely circulated throughout England and Wales and is now being used as a standard for good practice. The published report of the outbreak, and the hygiene code, has probably been a stimulus for the introduction or reintroduction of some local authority bills which require that tattooists, cosmetic piercers, and acupuncturists should register with their local authority and be subject to regular inspection.

Why standardise for birth weight in the analysis of perinatal mortality?

A. J. WIL COX, US National Institute of Environmental Health Sciences, I. T. RUSSELL, Medical Care Research Unit, University of Newcastle upon Tyne

In human populations, the chance of an infant surviving the perinatal period is closely related to its weight at birth. Consequently, when the effect on perinatal mortality of other variables is being considered, birth weight is often regarded as a confounding variable. Standardisation is a well-known method of neutralising the effects of confounding variables. Hence, many authors have been led to standardise for birth weight when analysing perinatal mortality.

Direct standardisation uses a common birth weight distribution to summarise differences in birth-weight-specific mortality rates. Indirect standardisation uses a common mortality curve to summarise differences in birth weight. Both methods are 
appropriate only if differences in mortality rates are consistent throughout the range of the confounding variable.

It is shown that this condition is rarely satisfied by birth-weight-specific mortality rates, and that standardisation is biased against groups with heavier birth weights. It is concluded that progress in adjusting for birth weight depends on the development of parametric models for birth weight and weight-specific mortality.

A comparison of the methods of standardised mortality ratios and regression models in life tables as used in industrial mortality studies

T. M. SORAHAN, Birmingham Regional Cancer Registry

The method of standardised mortality ratios (in which the mortality experience of the study cohort is compared with an expected experience based on the rates of mortality in some standard population) is the preferred method (i) to depict in general terms the mortality experience of a group of workers, (ii) to analyse data which include only a small number of deaths, (iii) to analyse data which include no suitable controls, or (iv) to analyse data which do not lend themselves to the testing of a specific hypothesis.

When data relating to the extent of exposure are available, however, the method of regression models in life tables, in which the exposures of those who have died are compared with those of the matching survivors in each year of follow-up, can be used to test a particular hypothesis.

Team care of the elderly in general practice J. R. STRANG, N. CAINE, Department of Community Medicine, University of Cambridge

In order to study multidisciplinary care of the elderly in the community, general practitioners and their nursing colleagues working in $\mathbf{1 7}$ practices completed a form after every contact with an elderly patient. Data collected over 14 days in each practice yielded forms for 6744 encounters with the elderly.

Doctors and community nurses were responsible for nine out of 10 contacts with the elderly. Only one in 100 encounters involved the health visitor. There were considerable interpractice variations in the contributions made by the various members of the primary care team. This was not easily explained by known patient or provider characteristics.

Intercommunication among professional groups was shown to be low, and there was little to support the view that teamwork is more likely to occur in group practices, or, conversely, that teamwork is hampered by inadequate practice accommodation. Less than $4 \%$ of the doctors' workload was initiated by other members of the team and in only $2 \%$ was there an intention to discuss the patient with other health workers.

Assessing the quality of medical care for children in Wessex. Delayed diagnosis of congenital dislocation of hip J. C. CA TFOR D, Wessex Regional Health Authority Congenital dislocation of the hip (CDH) is an important cause of physical disability in childhood. Since treatment by the age of one year substantially improves the prognosis, the
NHS has for the last 20 years sought to detect abnormalities early by the screening of all children in the newborn period.

Previous methods of assessing the effectiveness of surveillance programmes for $\mathrm{CDH}$ have failed to take sufficient account of variations in diagnostic criteria and severity. This can be overcome by evaluating the care of the most severe cases, those persistent over the age of one year who required hospital treatment. Such cases can easily be determined from Hospital Activity Analysis with the use of local diagnostic indexes.

In a feasibility study in Southampton, 86 cases of persistent $\mathrm{CDH}$ were determined; $31 \%$ were first diagnosed by an orthopaedic surgeon after 12 months of age, and $15 \%$ after 18 months of age. No statistically significant differences were found between the ability of hospital doctors and general practitioners to detect $\mathrm{CDH}$ in the neonatal period when those cases previously known to be at high risk (for example, breech delivery) were excluded. Of 43 'low risk' cases with no known predispositions, only $16 \%$ were detected neonatally.

\section{Drug use in Southampton hospitals}

A. H. HOUSTON, Wessex Regional Health Authority Lack of clinically useful information on drug costs and prescribing activities prompted a retrospective survey of drug use in over 5000 medical inpatients. The mean number of drugs per admission was four (range 0-28). Over 20\% of patients received no drugs, and the majority received eight or fewer. Patients' age, duration of admission, and the number of diagnoses accounted for over one-third of the total variability, with duration making the most important contribution and age the least. The majority of prescriptions were from a small number of therapeutic groups. Over $\mathbf{5 0 0}$ preparations were in use, but 154 were prescribed only once, and 10 drugs accounted for over one-third of all prescriptions.

Resource priorities in Kent : a Delphi study J. R. H. CHARLTON, D. I. PATRICK, G. MATHEWS, P. A. WEST, Department of Community Medicine, St. Thomas's Hospital Medical School, London

In 1979 , at the request of the Kent AHA, the spending priorities of everyone with some formal say in decision-making in Kent were studied.

The Delphi method was used, the central features of which are anonymity, controlled feedback, and statistical group summary. On each of three occasions, respondents were asked to estimate the relative importance of different service groups by allocating each $£ 1000$ of the NHS revenue budget between and within seven major service categories, as well as allocating money between capital and revenue, and substantial unplanned future revenue cuts and increases.

There proved to be consistent convergence over the three rounds, with evidence of groups influencing one another on certain issues. No one group dominated in all decisions. The method produced results broadly in line with the strategic plan, and achieved its aims, while also revealing to many participants the complexities of resource allocation under tight constraints. 